\title{
Multiprocessor task scheduling problem using hybrid discrete particle swarm optimization
}

\author{
T VAIRAM, S SARATHAMBEKAI* and K UMAMAHESWARI \\ Department of Information Technology, PSG College of Technology, Coimbatore 641004, India \\ e-mail: tvairam@gmail.com; vrs070708@gmail.com; uma.it@psgtech.ac.in
}

MS received 22 September 2015; revised 30 July 2018; accepted 14 September 2018; published online 20 November 2018

\begin{abstract}
Task Scheduling is a complex combinatorial optimization problem and known to be an NP hard. It is an important challenging issue in multiprocessor computing systems. Discrete Particle Swarm Optimization (DPSO) is a newly developed swarm intelligence technique for solving discrete optimization problems efficiently. In DPSO, each particle should limit its communication with the previous best solution and the best solutions of its neighbors. This learning restriction may reduce the diversity of the algorithm and also the possibility of occurring premature convergence problem. In order to address these issues, the proposed work presents a hybrid version of DPSO which is a combination of DPSO and Cyber Swarm Algorithm (CSA). The efficiency of the proposed algorithm is evaluated based on a set of benchmark instances and the performance criteria such as makespan, mean flow time and reliability cost.
\end{abstract}

Keywords. Cyber PSO; distributed systems; particle swarm optimization; swarm intelligence; task scheduling.

\section{Introduction}

Heterogeneous Multiprocessor System (HMS) is composed of computers with different speeds [1]. Task Scheduling (TS) is one of the key challenges in HMS. It can be classified into two categories based on the types of tasks [2]: tasks with no data dependencies (independent task) and tasks with precedence constraints (dependent task). In this paper, the scheduler schedules only the independent tasks in HMS. Independent tasks occur in many situations. For example, different users submit their jobs simultaneously to a super computer-center.

Heuristics are one of the suitable approaches to solve the TS problem in distributed systems. Some research works have been made in recent years using pure heuristics to discover near-optimal solutions [2,3]. These heuristics are fast, simple and easy to implement. To improve the quality of solutions, meta-heuristics have been presented for TS problem. The most popular meta-heuristic algorithms in the literature are Genetic Algorithm (GA), Differential Evolution (DE), Simulated Annealing (SA), Ant Colony Optimization (ACO) and Particle Swarm Optimization (PSO) [4].

PSO is a meta-heuristic algorithm proposed by Kennedy and Eberhart [1] in 1995, motivated by the flocking behavior of birds. This has been applied in wide area and different fields such as engineering, physics, mathematics

*For correspondence and chemistry. Task scheduling is the discrete optimization problems because it has discrete decision variables (e.g.: Task number or processor number). Discrete PSO (DPSO) was proposed to solve such kind of discrete problems efficiently $[5,6]$ because of discrete nature of the particles.

The performance of PSO greatly depends on its control parameters. Slightly different parameter settings may direct to different performance in the algorithm. Xin et al [7] presented Linearly Decreasing Inertia weight (LDI) for enhancing the efficiency and performance of PSO. Bansal et al [8] presented a comparative study on 15 strategies to set inertia weight in PSO. The four different modified PSO variants with respect to change of control parameters were addressed in TS problem [9]. Hamming inertia weight was proposed [10-12] to update the inertia weight in discrete domain. This weight was calculated based on hamming distance between the particles and the global best (leader particle) particle which ensures that the particles have not moved away from the leader particle. The proposed algorithm of this paper uses DPSO variant with hamming inertia weight to control the particles move only towards the leader particle of the swarm.

In PSO, a particle in the swarm should be interacted only with its previous best particle and the best particle of its neighbors. This is the main problem in PSO to restrict the learning capability of the particles. Peng et al [13] proposed Cyber Swarm Algorithm (CSA), which is an improved version of PSO. CSA allows particles to learn the knowledge from three leader particles such as previous best, the 
best particle of its neighbors and the historical best particles, which resulted in improved diversity. Peng et al [14] demonstrated the CSA for tackling the General Keyboard Arrangement Problem and also compared the efficiency of the CSA with standard PSO, Heuristic algorithm and Random algorithm. The cyber swarm is extended to solve multi-objective optimization problems namely Multi-Objective CSA (MOCSA). Peng and Ya-Tzu [15] illustrated the performance of MOCSA with NSGA-II and MOPSO.

The CSA algorithm [13-15] uses Global best (Gbest) model for the particle communication. In this model, all the particles will be attracted by the best particle found in the whole swarm. Therefore, the broadcasting of the best position and fitness information is very fast. This fast broadcasting may result in premature convergence (local optima) problem. The previous research work [16] presented Heap Intelligent DPSO (HIDPSO) approach which is based on Local best model (Lbest) for reducing the premature convergence problem in scheduling of metatasks in distributed systems. HIDPSO allows the particle to learn the knowledge only from two best positions such as Pbest and Lbest. This learning restriction may reduce the diversity of the algorithm. To address this issue, this paper presents the hybridization of HIDPSO with CSA namely Cyber HIDPSO (CHIDPSO) which will improve the diversity of the algorithm.

The remainder of the paper is organized as follows. Section 2 describes the problem formulation. The proposed algorithm is presented in section 3. Experimental results are reported in section 4 . Section 5 presents the computational complexity of the algorithm. Finally, the conclusions are provided in section 6 .

\section{Problem formulation}

\subsection{Task model}

A Heterogeneous Computing (HC) system consists of a number of heterogeneous Processor Elements (PEs) connected with a mesh topology. Let $T=\left\{T_{1}, T_{2} \ldots, T_{n}\right\}$ denote the $n$ number of tasks that are independent of each other to be scheduled on $\mathrm{m}$ processors $\mathrm{P}=\left\{\mathrm{P}_{1}, \mathrm{P}_{2} \ldots, \mathrm{P}_{\mathrm{m}}\right\}$. Because of the heterogeneous nature of the processors and disparate nature of the tasks, the expected execution times of a task executing on different processors are different.

Every task has an Expected Time to Compute (ETC) on a specific processor. The ETC values are assumed to be known in advance. An ETC matrix is $\mathrm{n} x \mathrm{~m}$ matrix in which $\mathrm{m}$ is the number of processors and $\mathrm{n}$ is the number of tasks. One row of the ETC matrix represents estimated execution time for a specified task on each PE. Similarly, one column of the ETC matrix consists of the estimated execution time of a specified PE for each task.

The TS problem is formulated based on the following assumptions:
- All tasks are available at zero time. Processors are always available.

- The execution time of each task on each processor is known and constant.

- Preemption is not allowed. Each processor can process only one task at a time.

- A task cannot be processed on more than one processor at a time.

- Each processor uses the First-Come, First-Served (FCFS) method for performing the received tasks.

\subsection{Scheduler model}

A static scheduler model in distributed systems is shown in figure 1. The scheduler manages two queues such as Task Queue (TQ) and Processor Queue (PQ). The scheduling algorithm in the central scheduler is started to work with TQ. TQ contains a set of tasks in a particle. The scheduler is responsible for distributing each task in the TQ to the individual PQ based on the workload of the each processor in the distributed systems. Once the scheduler completes to place all the tasks from the TQ to $\mathrm{PQ}$, the processors will start executing the tasks in their own PQ.

\section{The proposed CHIDPSO algorithm}

The CHIDPSO incorporates the heuristic based swarm initialization, heap tree based particle communication and reference set in velocity update method for reducing premature convergence and improving the diversity of the algorithm. The complete flow of the proposed work is given in figure 2.

The following subsections describe in detail the steps involved in the CHIDPSO algorithm.

\subsection{Swarm initialization}

Swarm Initialization plays an important role in the performance of the CHIDPSO algorithms. IDPSO [17] algorithm was proposed in the previous work which improved the quality of the initial seed solution with the incorporation of heuristics such as Open Path greedy Opposite (OPO) and

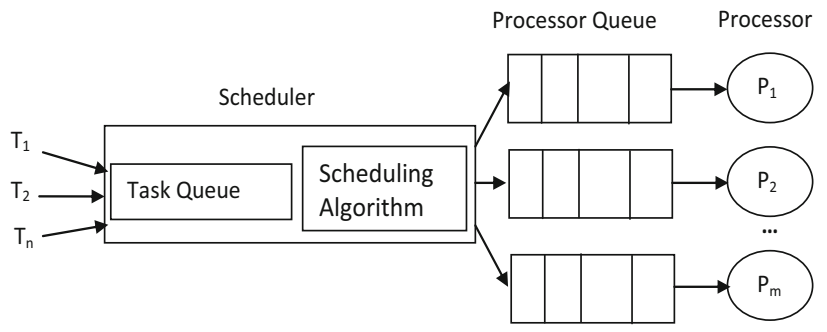

Figure 1. Scheduling model for heterogeneous environment. 


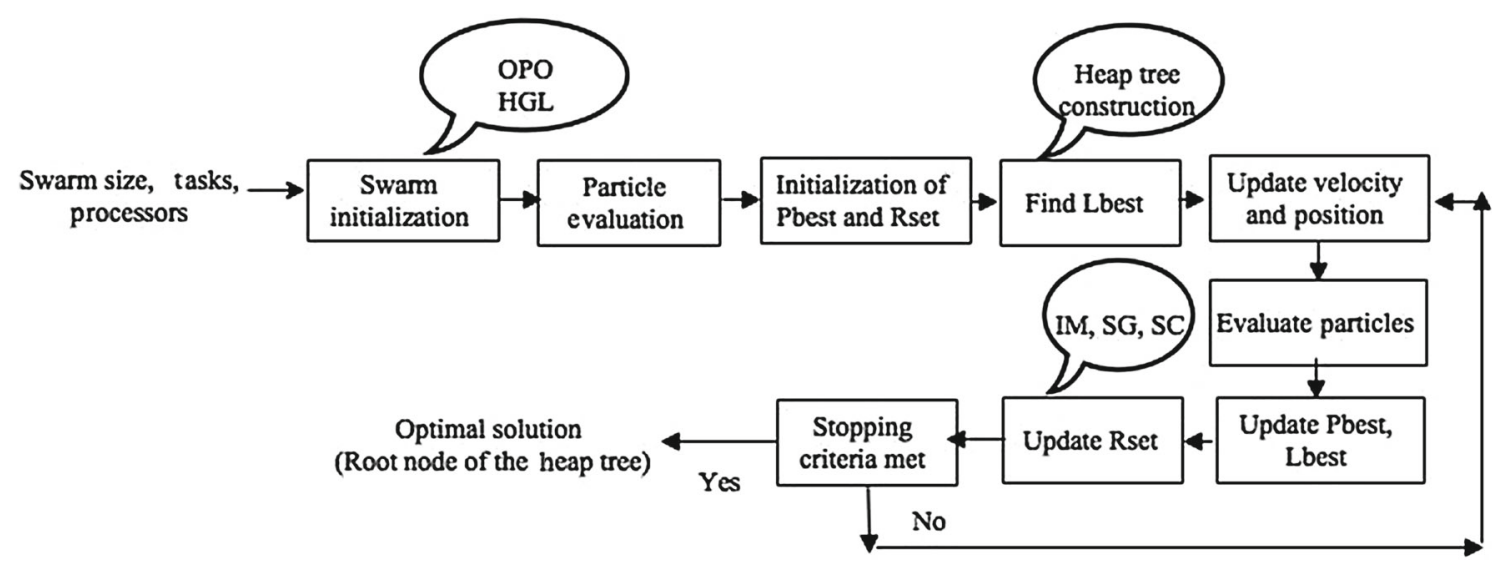

Figure 2. The flow diagram of CHIDPSO algorithm. *Where OPO - Open Path greedy Opposite; HGL - Heuristic based Greedy static Load balancing algorithm; Rset - Reference set; IM - Improvement Method (IM); SG - Subset Generation; SC - Solution Combination (SC).

Heuristic based Greedy Load balancing (HGL) in swarm initialization. Therefore, CHIDPSO uses OPO and HGL for swarm initialization to improve initial quality of the solutions.

\subsection{Particle evaluation}

In CHIDPSO, the particles are evaluated using the three performance metrics such as makespan, mean flow time and reliability cost.

3.2a Makespan: Makespan (MS) computes the throughput of the distributed systems, assuming $\mathrm{C}_{\mathrm{ij}}(\mathrm{i} \varepsilon\{1,2, \ldots, \mathrm{n}\}, \mathrm{j} \varepsilon\{1,2, \ldots, \mathrm{m}\})$ is the execution time for performing $\mathrm{i}^{\text {th }}$ task in $\mathrm{j}^{\text {th }}$ processor and $\mathrm{W}_{\mathrm{j}}(\mathrm{j} \varepsilon\{1,2, \ldots, \mathrm{m}\})$ is the previous workload of $\mathrm{P}_{\mathrm{j}}$. According to the above definition, makespan can be estimated using Eq. (1):

$$
\mathrm{MS}=\max \left\{\sum_{\mathrm{ij}} \mathrm{C}_{\mathrm{ij}}+\mathrm{W}_{\mathrm{j}}\right\} \mathrm{j} \varepsilon(1,2,3 \ldots, \mathrm{m})
$$

3.2b Mean flow time: Flow time measures the Quality of Service of the distributed system. The value of Mean Flow Time (MFT) is used to evaluate the flow time. Assume $\mathrm{k}$ to be the total number of tasks assigned to processor $P_{i}$ and $F_{j i}$ the finishing time of task $T_{j}$ on a processor $P_{i}$,

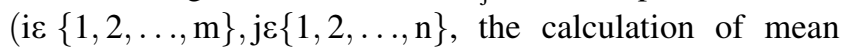
flow time are given in Eqs. (2) and (3).

$$
\begin{gathered}
\text { MFT }=\frac{\sum_{\mathrm{i}=1}^{\mathrm{m}} \text { M_Flow } i}{\mathrm{~m}} \\
\mathrm{M}_{-} \text {Flow }_{\mathrm{i}}=\frac{\sum_{\mathrm{j}=1}^{\mathrm{k}} \mathrm{F}_{\mathrm{ji}}}{\mathrm{k}_{\mathrm{i}}}
\end{gathered}
$$

3.2c Reliability cost: Reliability Cost (RC) [18] is the indicator of how reliable a given system is when a group of tasks are assigned to it. It is indirectly proportional to reliability. RC is the summation of processor reliability and link reliability. Here, the tasks are independent of each other and there is no communication link between tasks. Therefore, link reliability is set to zero. In processor reliability, processor failures are assumed to be independent and it follows a Poisson process with a constant failure rate. The RC is defined in Eq. (4), where $X\left(T_{i}\right)=j$ indicates that task $T_{i}$ is allocated to $P_{j}$ and $\lambda_{j}$ is the failure rate of processor $P_{j}$.

$$
\mathrm{RC}=\sum_{j=1}^{m} \sum_{X\left(T_{i}\right)=j} \lambda_{j} C_{i j}\left(T_{i}\right)
$$

3.2d Fitness function: Fitness function is used to test the quality of the solution. Since it is a minimization problem, a better solution has the lower fitness value. The fitness function comprises tri-objectives such as MS, MFT and RC. This can be estimated using Eq. (5), where the weights $\mathrm{W}_{1}, \mathrm{~W}_{2}$ and $\mathrm{W}_{3}$ indicate the significance of the objectives in meta-task scheduling problem.

$$
\begin{aligned}
\text { Fitness }= & \mathrm{W}_{1} \text { Makespan }+\mathrm{W}_{2} \text { Mean Flow Time } \\
& +\mathrm{W}_{3} \text { Reliability Cost }
\end{aligned}
$$

The HIDPSO [16] experimented the fitness function with different weight values and concluded that $0.4,0.4$ and 0.2 for the weights $\mathrm{W}_{1}, \mathrm{~W}_{2}$ and $\mathrm{W}_{3}$, respectively provided better results in the meta-task scheduling problem. Therefore, the CHIDPSO follows the same fitness function for evaluating the quality of the particles.

\subsection{Update the particle's Personal best and local best position}

The Pbest is the personal best position visited so far by a particle. The local best (Lbest) of the particle can be obtained through the Binary Heap Tree (BHT) based communication with its neighboring particles [16]. 
In CHIDPSO algorithm, the learning of the particles is not limited to communication with the Pbest and Lbest. And also, the learning involves particles from a dynamically maintained reference set. This learning knowledge of CHIDPSO improves the diversity of the swarm. The next subsection describes in detail about the reference set.

\subsection{Reference set initialization and updation}

The reference set consists of a mixture of historical best solutions and diverse solutions. This would improve the quality of the solutions and diversity of the algorithm, respectively. It is represented by $\operatorname{refSet}[\mathrm{i}], \mathrm{i}=1 \ldots$ REFSIZE, where REFSIZE is the size of the reference set. Generally, the REFSIZE is five times lesser than the swarm size [13].

3.4a Reference set initialization: Reference set initialization is the process of selecting the initial particles for reference set. This is performed using the diversification generation method of Scatter Search (SS) [19]. Using this method, the proposed algorithm fills the reference set with $\mathrm{S} 1$ number of quality solution and S2 number of diverse solutions, which is explained as follows:

- Choose the best S1 number of particles from Pbest array with size $\mathrm{N}$ (This is known as the quality solution)

- Calculate S2 number of opposite particles of the selected S1 particles using OPO technique. (This is known as the diverse solution, therefore, $\mathrm{REFSIZE}=\mathrm{S} 1+\mathrm{S} 2$ ).

The initialization is done only in the first iteration before updating the particle's velocity. The pseudo code for initializing the reference set is given in figure 3 . This initialization process invokes the function Function 1 for populating the solutions in the reference set. This function code is presented in figure 4.

\begin{tabular}{|l|}
\hline Pseudo code 1: Reference set Initialization \\
\hline Input: Pbestparticles with size N; Output: refSet with size N/5 \\
\hline begin \\
Arrange the Pbest particles in ascending order based on the Pbest fitness (PFit). \\
// Invoke Function 1 to create an initial refSet with size N/5. \\
Call Reference set solution selection (in Pbest, in N, in PFit, Out refSet). \\
end \\
\hline
\end{tabular}

Figure 3. Initialization of reference set.

\begin{tabular}{|c|}
\hline Function 1: Reference set solution selection \\
\hline Input: ParticleSet, ParticleSet_Size,Fitness; Output: refSet with REFSIZE \\
\hline 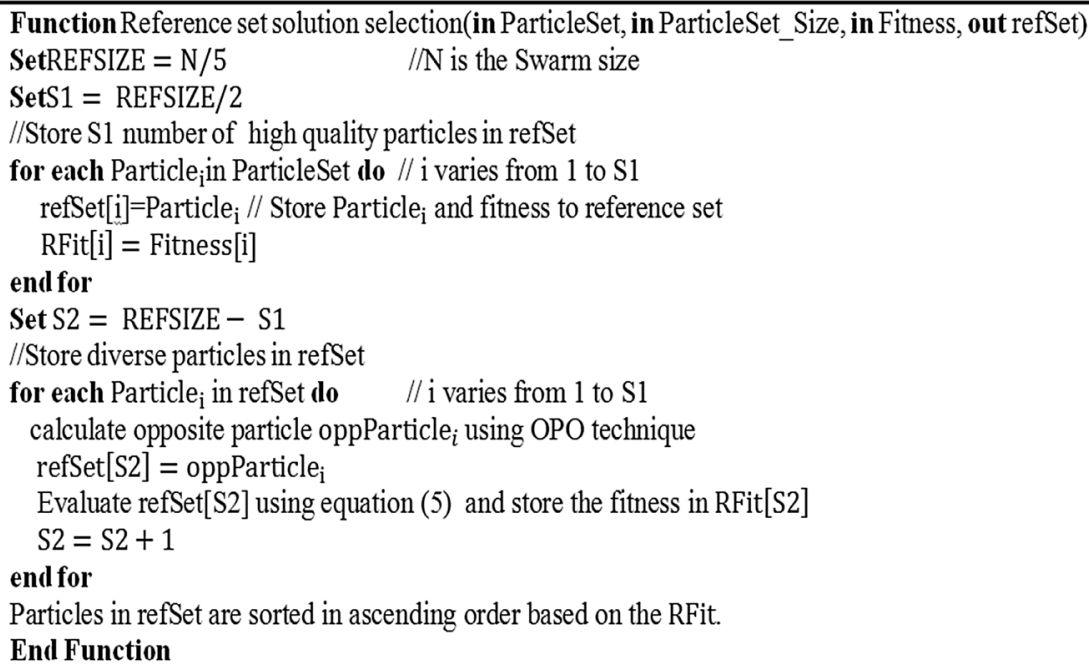 \\
\hline
\end{tabular}

Figure 4. Particles selection for the Reference set. 
Figures 3 and 4 include the direction parameters in the pseudo code such as in and out, which indicate input to the function and output from the function, respectively. The reference set particles will get updated after the first iteration. The high quality and low quality particles will be selected from the reference set for updating the particles. Therefore, the particles in the reference set are arranged according to the quality of the particles such that the refSet [1] contains the high quality particle (least fitness) and refSet [REFSIZE] contains the less quality particle (highest fitness).

3.4b Reference set updation: Reference set is updated using Improvement Method (IM), Subset Generation (SG) and Solution Combination (SC) methods of Scatter Search (SS) algorithm.

The Improvement Method performs like a selection operation of Genetic Algorithm. The SG method generates subsets from the reference set. Since generating all the subsets from the reference set is a time consuming process, in order to reduce the time complexity of SG, IM is required to select the limited particles from the reference set for subset generation. Figure 5 depicts the procedure for the IM. Here, the IM selects two best particles and one worst particle from refSet to build selection set for SG.
The Subset Generation method generates subsets from the selected particles in reference set. It is categorized into three main types namely Sub type 1, Sub type 2 and Sub type 3 . The experiments of the proposed algorithm with different subset types (1, 2, and 3 ) are conducted. The preliminary experiments shows that the subset of type 2 and 3 have a small probability of getting new better solutions, this may because our diversification generation module generates the diverse solutions using OPO heuristic. Therefore, only the subset of type 1 is adopted for this problem. Generally, the sub type 1 method is preferred to perform SG due to its simplicity and lesser time complexity when compared to other sub types [13]. Figure 6 shows the pseudo code for the subset generation method. This method invokes a function Function 2, which is given in figure 8 . Function 2 generates the particles from the three subset combinations such as $\{$ (best 1 , best 2$)\},\{$ (best1, worst) $\}$, \{(best2, worst) $\}$ based on the sub type 1 generation method of SG. An illustrative example of SG is given in figure 7.

From figure 7 , it is evident that a single swap will provide two new particles and it requires 2 transfers (one in best 1 and another in best 2). If the particle size is $n$, then the SG method will perform $n$ number of swaps and $2^{\text {n }}$ number of transfers. Finally, it will produce $2^{\mathrm{n}}$ number of solutions. In order to reduce the number of swaps and

\begin{tabular}{|l|}
\hline Pseudo code2:Improvement method \\
\hline Input: refSet with REFSIZE; Output: SelectionSet with size 3 \\
\hline //Restrict the size of the SelectionSet so as to reduce the time complexity. \\
begin \\
//Store two best particles and one worst particle in Selectionset \\
SelectionSet[1] = refSet[1] \\
SelectionSet[2] = refSet[2] $/ / 1^{\text {st }}$ best (best1) \\
SelectionSet[3] $=\operatorname{refSet[REFSIZE]~}$ \\
end
\end{tabular}

Figure 5. Procedure for improvement method in SS.

\begin{tabular}{|l|}
\hline Pseudo code3:Subset Generation Method \\
\hline Input: SelectionSet with size 3 ; \\
Output: subSet1, subSet2 and subSet3 with each of size REFSIZE \\
\hline begin \\
$\quad$ call Setgeneration (in SelectionSet[1], in SelectionSet[2], outsubSet1) \\
call Setgeneration (in SelectionSet[1], in SelectionSet[3], out subSet2) \\
call Setgeneration (in SelectionSet[2], in SelectionSet[3], outsubSet3) \\
end
\end{tabular}

Figure 6. Subset generation method. 


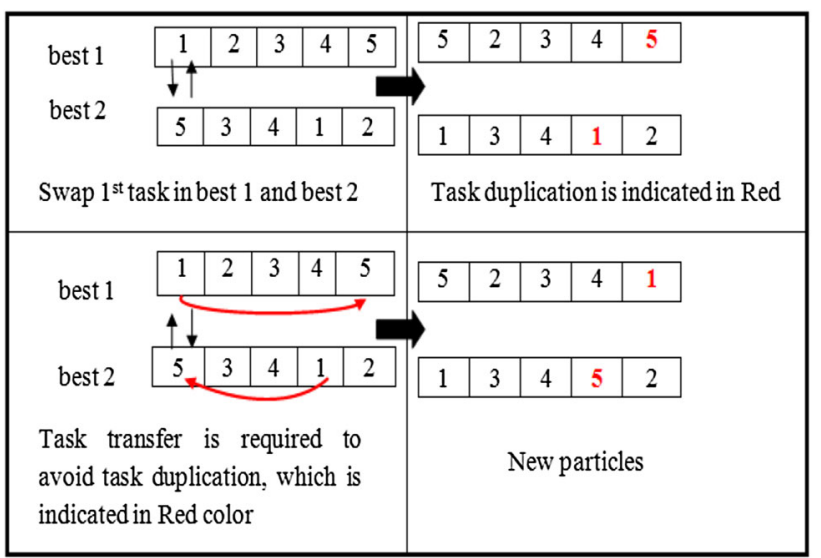

Figure 7. Sample for the SG of $\{$ best 1 , best 2$\}$.

number of transfers, the SG method does not consider all the tasks for the swap. Instead of performing all the swap operations, it randomly selects the tasks for performing swap operation to generate the new solutions, as explained in figure 8.

Solution Combination method is the last phase of reference set updation. Figure 9 explains the procedure to perform the SG method. During this phase, all the generated subsets (subSet1, subSet2 and subSet3) in SG are combined to make SolnCombine with REFSIZE $* 4$ number of particles. This is referred by the Eq. (6), where U performs to combine the particles from refSet, subSet1, subSet2 and subSet3 into single solution pool SolnCombine.

$$
\begin{aligned}
\text { SolnCombine }= & \text { refSet } \cup \text { subSet } 1 \cup \text { subSet2 } \\
& \cup \text { subSet } 3
\end{aligned}
$$

In CHIDPSO, each particle is having three leader particles such as Pbest, Lbest and systematically choose each member from the reference set as the third leader. Equation (7) is used to calculate the reference particle RefParticle $_{i}$ of a Particle ${ }_{\mathrm{i}}$ in the swarm with size N, where i varies from 1 to $\mathrm{N}$. The three leader particles are updated before updating the velocity and position of the particle.

$$
\text { RefParticle }_{i}=\mathrm{i} \bmod \text { REFSIZE }
$$

The following subsection describes the way to update the velocity and position of the particles in the swarm.

\subsection{Updating the particle's velocity and position}

The velocity of the particle is redefined to operate it in a discrete domain directly [12]. This velocity has four parts: old velocity, learning from Pbest, Lbest and Rset. This is calculated using Eq. (8). Here, the operators: U performs

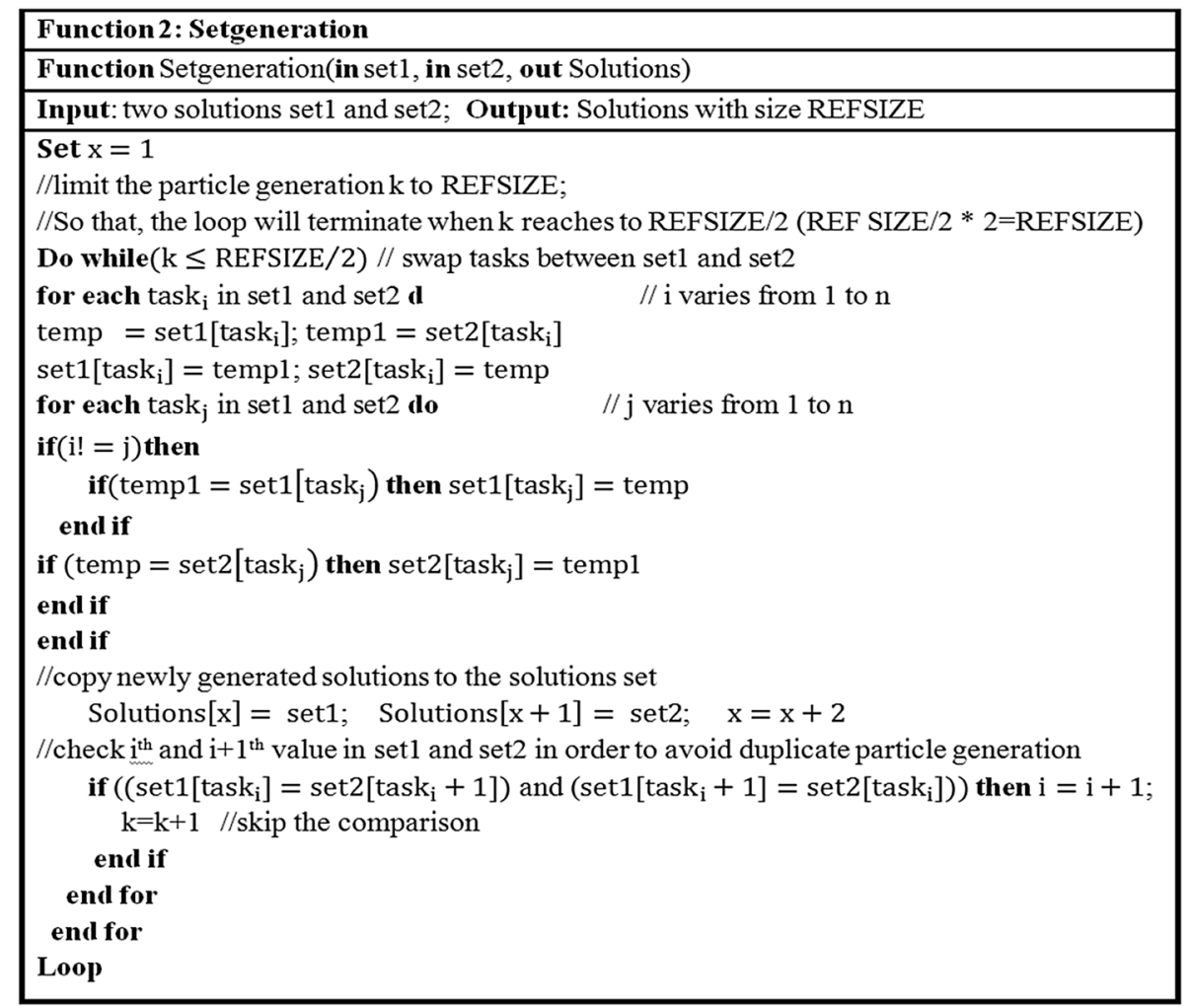

Figure 8. Set generations for the SG method. 


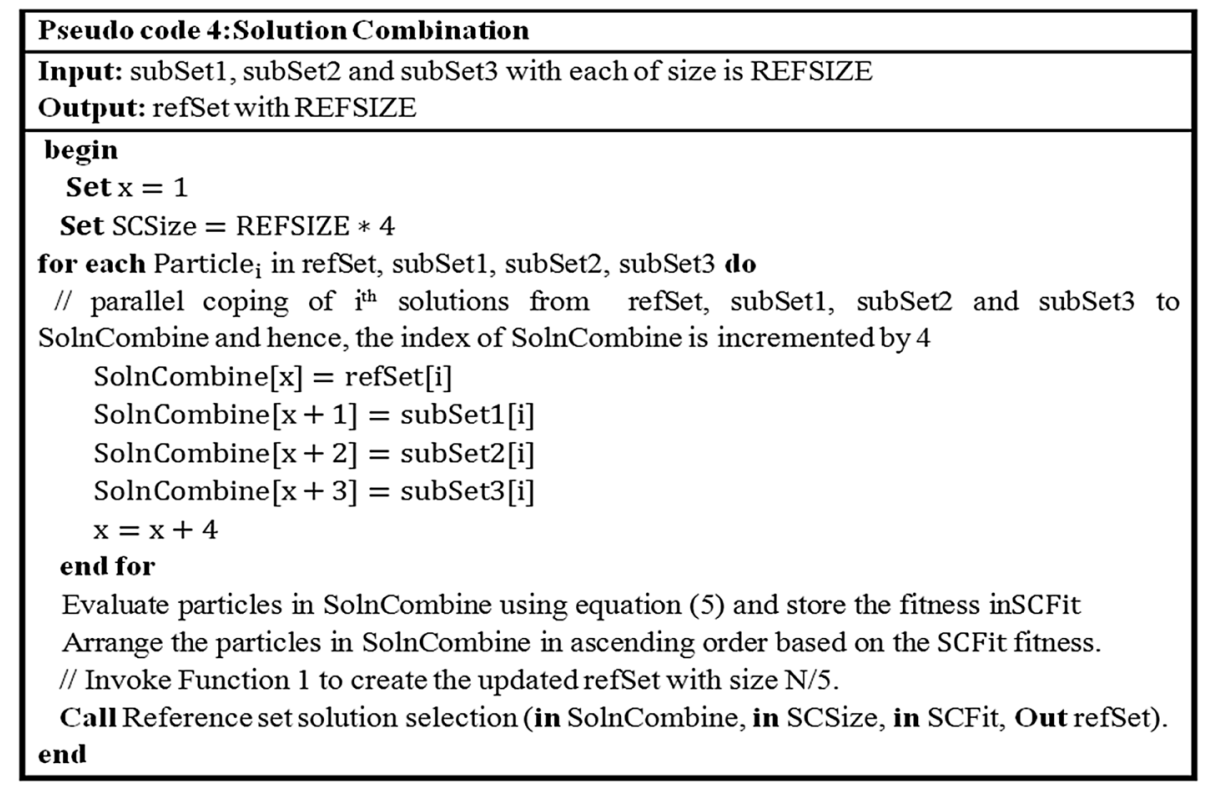

Figure 9. Procedure for the solution combination method.

merging operation and "Swap" represents the swapping sequence of the operands. The four coefficients: $\mathrm{W}, \mathrm{C}_{1} \mathrm{R}_{1}$, $\mathrm{C}_{2} \mathrm{R}_{2}$ and $\mathrm{C}_{3} \mathrm{R}_{3}$ represent the importance of old velocity, particle's Pbest, neighborhood best and reference set, respectively. In this paper, the learning factors $\mathrm{C}_{1}, \mathrm{C}_{2}$ and $\mathrm{C}_{3}$ are set to 1 .

$$
\mathrm{V}_{\mathrm{i}}^{(\mathrm{t}+1)}(\mathrm{j})=\mathrm{A} \mathrm{U} \mathrm{B} \mathrm{U} \mathrm{C} \mathrm{U} \mathrm{D}
$$

Where

$$
\begin{gathered}
\mathrm{A}=\mathrm{WV}_{\mathrm{i}}^{\mathrm{t}}(\mathrm{j}) \\
\mathrm{B}=\mathrm{C}_{1} \mathrm{R}_{1}\left(\operatorname{Pbest}_{\mathrm{i}}^{\mathrm{t}}(\mathrm{j}) \operatorname{Swap}_{\left.\operatorname{present}_{\mathrm{i}}^{\mathrm{t}}(\mathrm{j})\right)}\right. \\
\mathrm{C}=\mathrm{C}_{2} \mathrm{R}_{2}\left(\operatorname{Lbest}^{\mathrm{t}} \operatorname{Swap} \operatorname{present}_{\mathrm{i}}^{\mathrm{t}}(\mathrm{j})\right) \\
\mathrm{D}=\mathrm{C}_{3} \mathrm{R}_{3}\left(\operatorname{Rset}^{\mathrm{t}} \operatorname{Swap} \operatorname{present}_{\mathrm{i}}^{\mathrm{t}}(\mathrm{j})\right)
\end{gathered}
$$

The CHIDPSO uses hamming distance based inertia. Moving the particles based on the hamming distance between the particles and their Local best that ensures that the particles have not moved away from the Lbest [12]. The Hamming inertia weight is calculated using Eq. (13), where $\mathrm{W}_{0}$ is the random number between 0.5 and $1, \mathrm{H}_{\mathrm{i}}$ is the current hamming distance of $i^{\text {th }}$ particle from the Lbest and $\mathrm{MDH}$ is the maximum distance of a particle from the Lbest in that generation. The inertia weight $(W)$ in Eq. (8) is calculated using Eq. (13).

$$
\begin{gathered}
\mathrm{W}=\mathrm{W}_{0}\left(1-\frac{\mathrm{H}_{\mathrm{i}}}{\mathrm{MDH}}\right) \\
\mathrm{H}_{\mathrm{i}}=\text { Hamming distance }\left(\text { Gbest }^{\mathrm{t}} \text {, } \operatorname{present}_{\mathrm{i}}^{\mathrm{t}}\right)
\end{gathered}
$$

$$
\mathrm{MDH}=\operatorname{Max}\left(\mathrm{H}_{\mathrm{i}}\right)
$$

$\mathrm{R}_{1}, \mathrm{R}_{2}$ and $\mathrm{R}_{3}$ in Eqs. (10), (11) and (12) are random numbers between 0 and 1 . If three random numbers $\left(R_{1}, R_{2}\right.$ and $R_{3}$ ) are independent then the algorithm will face the following problems:

- Three random parameters are high: Pbest and social experiences (Lbest, Rset) are extremely used and the particle is moved too far away from the local optimum.

- Three random parameters are low: Pbest and social experiences (Lbest, Rset) are not used completely and the convergence speed is very low.

In order to address the above stated problems, CHIDPSO makes the dependency among the three random parameters to balance the personal and social experiences of the particle. In this method, one single random number $R_{1}$ is chosen then $R_{2}$ and $R_{3}$ are generated based on $R_{1}$. Equations (16), (17) and (18) present the way to create a dependency among the three random numbers, where random $(\mathrm{N})$ generates a random number between 0 and $\mathrm{N}, \mathrm{N}$ is the swarm size.

$$
\begin{gathered}
\mathrm{R}_{1}(\mathrm{t})=\operatorname{random}(\mathrm{N}) / \mathrm{N} \\
\mathrm{R}_{2}(\mathrm{t})=\left(1.0-\mathrm{R}_{1}(\mathrm{t})\right) \operatorname{random}(\mathrm{N}) / \mathrm{N} \\
\mathrm{R}_{3}(\mathrm{t})=1.0-\mathrm{R}_{1}(\mathrm{t})-\mathrm{R}_{2}(\mathrm{t})
\end{gathered}
$$

After updating the velocity, update the position of the particle using Eq. (19).

$$
\operatorname{present}_{\mathrm{i}}^{(\mathrm{t}+1)}(\mathrm{j})=\operatorname{present}_{\mathrm{i}}^{\mathrm{t}}(\mathrm{j})(\operatorname{swap}) \mathrm{V}_{\mathrm{i}}^{(\mathrm{t}+1)}(\mathrm{j})
$$




\subsection{Stopping condition}

The above iterative processes on a swarm will continue until a pre-defined maximum number of iterations have been reached or no change in the fitness value for consecutive iterations.

The average Relative Percentage Deviation (RPD) [5] is also calculated along with makespan, mean flow time and reliability cost for comparing the efficiency of the algorithms. It is calculated using Eq. (20), where $\mathrm{P}$ is the average result of the CHIDPSO algorithm and $\mathrm{AC}_{\mathrm{i}}$ is the average result provided by other algorithms.

$$
\mathrm{RPD}=\left(\mathrm{AC}_{\mathrm{i}}-\mathrm{P}\right) / \mathrm{P} * 100
$$

\section{Simulation results and analysis}

The simulation results are attained using a set of benchmark ETC instances [20] for the distributed heterogeneous systems. The algorithms are coded in Java and executed in Net Beans IDE.

\subsection{Benchmark instances description}

The simulation is performed on the benchmark ETC instances [20] which are categorized in 12 types of ETC's based on three metrics: task heterogeneity, machine heterogeneity and consistency [5].

All instances consisting of 512 tasks and 16 processors are classified into 12 different types of ETC matrices according to the above 3 metrics.

The instances are labeled as a_b_c as follows:

- a shows the type of consistency; c - consistent, i inconsistent, and $\mathrm{s}-$ semi-consistent.
- $\mathrm{b}$ indicates the heterogeneity of the tasks; hi - high and lo - low.

- c represents the heterogeneity of the machines; hi high and lo - low.

\subsection{Parameter set-up}

The following parameters are initialized for simulating the algorithms.

- Population size: 50

- Number of iteration: 500

- The Failure rate for each processor is uniformly distributed [18] in the range from $0.95 \times 10^{-6} / \mathrm{h}$ to $1.05 \times 10^{-6} / \mathrm{h}$.

The algorithms are stochastic based algorithms. Each independent run of the same algorithm on a particular problem instance may yield a different result. To make a better comparison of the algorithms, each experiment was repeated 10 times with different random seeds and average of the results are tabulated and presented.

\subsection{Performance comparisons}

Table 1 presents the comparison of objective values of the proposed CHIDPSO and the existing CSA algorithms.

From table 1, it can be observed that the heuristic based initialization and dynamic BHT based particle communication in CHIDPSO provided better results in most of the ETC instances when compared to the existing CSA algorithm. Compared to the existing CSA, the overall average performance improvement of CHIDPSO algorithm in terms of makespan is $75.65 \%$, mean flow time is $85.36 \%$ and reliability cost is $32.11 \%$ across all ETC instances.

Table 1. Performance comparison of CHIDPSO with existing CSA.

\begin{tabular}{|c|c|c|c|c|c|c|c|c|c|}
\hline \multirow[b]{2}{*}{ ETC instances } & \multicolumn{3}{|c|}{ Makespan } & \multicolumn{3}{|c|}{ Mean flow time } & \multicolumn{3}{|c|}{ Reliability cost } \\
\hline & CHIDPSO & CSA & RPD & CHIDPSO & CSA & RPD & CHIDPSO & CSA & RPD \\
\hline c_lo_lo & 13123.42 & 31105.15 & 137.02 & 6140.413 & 7360.07 & 19.86 & 0.186019 & 0.139401 & -25.06 \\
\hline c_lo_hi & 25201.01 & 31207.12 & 23.83 & 11638.44 & 35010.61 & 200.81 & 0.460741 & 0.440998 & -4.28 \\
\hline c_hi_lo & 11096.34 & 35057.91 & 215.94 & 5962.06 & 7505.41 & 25.88 & 0.181204 & 0.225683 & 24.54 \\
\hline c_hi_hi & 17103.05 & 20207.41 & 18.15 & 12002.87 & 24217.12 & 101.76 & 0.464482 & 0.489956 & 5.48 \\
\hline i_lo_lo & 25101.27 & 32036.61 & 27.62 & 13162.99 & 22990.95 & 74.66 & 0.305049 & 0.440408 & 44.37 \\
\hline i_lo_hi & 28056.91 & 32017.38 & 14.11 & 14008.09 & 23199.49 & 65.61 & 0.434033 & 0.490009 & 12.89 \\
\hline i_hi_lo & 23072.86 & 34825.46 & 50.93 & 11322.52 & 21491.42 & 89.81 & 0.393784 & 0.429941 & 9.18 \\
\hline i_hi_hi & 28045.24 & 35197.95 & 25.5 & 12072.92 & 21000.93 & 73.95 & 0.423777 & 0.475999 & 12.32 \\
\hline s_lo_lo & 23106.64 & 44112.13 & 90.91 & 10000.69 & 20911.97 & 109.11 & 0.237494 & 0.469001 & 97.47 \\
\hline s_lo_hi & 28002.32 & 36512.33 & 30.39 & 11163.52 & 22001.01 & 97.07 & 0.427074 & 0.490001 & 14.73 \\
\hline s_hi_lo & 12015.36 & 41003.04 & 241.25 & 6016.54 & 7399.16 & 22.98 & 0.168483 & 0.420964 & 149.85 \\
\hline s_hi_hi & 28053.28 & 37074.41 & 32.15 & 12732.82 & 30919.06 & 142.82 & 0.340823 & 0.489941 & 43.75 \\
\hline Average & & & 75.65 & & & 85.36 & & & 32.11 \\
\hline
\end{tabular}


The resource utilization [21] is the performance criterion for the scheduler to perform scheduling with balancing the load. The processor's utilization is defined as the percentage of time that processor $P_{j}$ is busy during the scheduling time. The processor's utilization $P U_{j}$ for the processor $P_{j}$ is calculated using Eq. (21).

$$
\mathrm{PU}_{\mathrm{j}}=\operatorname{Aval}\left(\mathrm{P}_{\mathrm{j}}\right) / \text { Makespan for } \mathrm{j}=1 \ldots \mathrm{m}
$$

Where, $\operatorname{Aval}\left(P_{j}\right)$ is the processor's availability time. The processor availability time is the time when the processor $P_{j}$ completes the execution of all the assigned tasks.

The resource utilization $R U$ is the average of processor's utilization, which is calculated using Eq. (22).

$$
\mathrm{RU}=\frac{\sum_{\mathrm{j}=1}^{\mathrm{m}} \mathrm{PU}_{\mathrm{j}}}{\mathrm{m}}
$$

Resource Utilization (RU) of CHIDPSO and CSA is calculated using Eq. (22) and the comparison of performance is presented in figure 10 .

From figure 10, it can be inferred that the RU of CSA is varying from 0.5 to 0.9 and the proposed CHIDPSO is

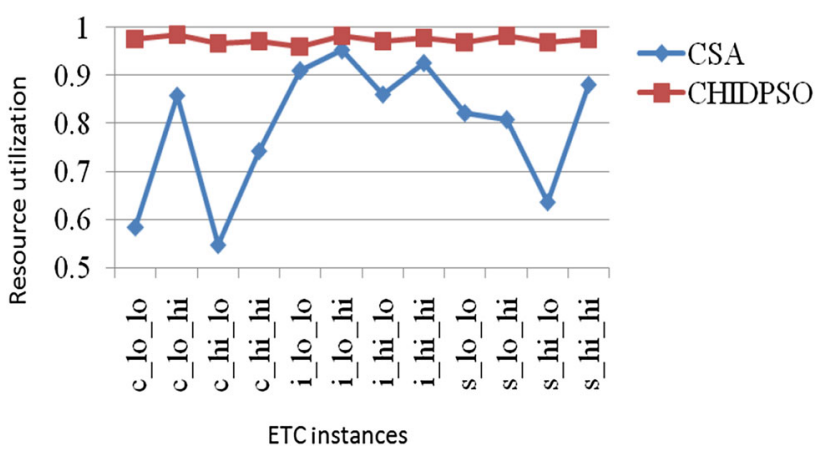

Figure 10. CHIDPSO Vs CSA with respect to RU. between 0.95 and 1.0 with respect to different ETC instances. The average percentage of RU of CSA is $79.39 \%$ and CHIDPSO is $97.43 \%$ across all ETC instances. Due to HGL load balancing heuristic in CHIDPSO, this algorithm utilized the resources efficiently than the existing CSA algorithm nearly more than $18 \%$.

Table 2 presents the comparison of objective values of the proposed CHIDPSO and the previously proposed HIDPSO algorithms [16]. The multi-objective values makespan, mean flow time and reliability cost presented in table 2 show that the CHIDPSO gives better objective values than HIDPSO in most of the ETC instances due to the inclusion of the reference set.

From table 2, it can be observed that the reference set in CHIDPSO provided better results in most of the ETC instances when compared to the HIDPSO. Compared to HIDPSO, the overall average performance improvement of CHIDPSO algorithm in terms of makespan is $10.28 \%$, mean flow time is $6.83 \%$ and reliability cost is $5.61 \%$ across all ETC instances.

The multi-objective optimization problems can be broadly solved using Aggregating approach and Pareto dominance approach. From literature, the NSGA-II and NSPSO are well-known Pareto dominance based algorithms for meta-task scheduling problem in distributed systems. The NSHIDPSO [22, 23] performed better in terms of makespan is $52.12 \%$, mean flow time is $24.03 \%$ and reliability cost is $30.43 \%$ when compared to the existing NSPSO across all ETC instances. Compared to the existing NSGA-II, the NSHIDPSO provided better performance in terms of makespan is $54.22 \%$, mean flow time is $61.39 \%$ and reliability cost is $31.89 \%$ across all ETC instances. Table 3 presents the performance comparison of CHIDPSO with NSHIDPSO.

The CHIDPSO uses aggregating approach for prioritizing the objectives based on their significance in the metatask scheduling problem. This type of prioritization of

\begin{tabular}{|c|c|c|c|c|c|c|c|c|c|}
\hline \multirow[b]{2}{*}{ ETC Instances } & \multicolumn{3}{|c|}{ Makespan } & \multicolumn{3}{|c|}{ Mean Flow Time } & \multicolumn{3}{|c|}{ Reliability Cost } \\
\hline & HIDPSO & CHIDPSO & RPD & HIDPSO & CHIDPSO & RPD & HIDPSO & CHIDPSO & RPD \\
\hline c_lo_lo & 13178.05 & 13123.42 & 0.41 & 6449.38 & 6140.413 & 5.03 & 0.190886 & 0.186019 & 2.61 \\
\hline c_lo_hi & 27724.31 & 25201.01 & 10.01 & 13028.21 & 11638.44 & 11.94 & 0.462184 & 0.460741 & 0.31 \\
\hline c_hi_lo & 13059.35 & 11096.34 & 17.69 & 6205.41 & 5962.06 & 4.08 & 0.192498 & 0.181204 & 6.23 \\
\hline c_hi_hi & 20220.51 & 17103.05 & 18.22 & 13075.56 & 12002.87 & 8.93 & 0.466072 & 0.464482 & 0.34 \\
\hline i_lo_lo & 27581.99 & 25101.27 & 9.88 & 13127.56 & 13162.99 & -0.26 & 0.300782 & 0.305049 & -1.39 \\
\hline i_lo_hi & 30292.47 & 28056.91 & 7.96 & 14039.73 & 14008.09 & 0.22 & 0.440281 & 0.434033 & 1.43 \\
\hline i_hi_lo & 25617.19 & 23072.86 & 11.02 & 12531.76 & 11322.52 & 10.67 & 0.400137 & 0.393784 & 1.61 \\
\hline i_hi_hi & 30074.69 & 28045.24 & 7.23 & 14235.47 & 12072.92 & 17.91 & 0.440129 & 0.423777 & 3.85 \\
\hline s_lo_lo & 25275.83 & 23106.64 & 9.38 & 10008.87 & 10000.69 & 0.08 & 0.282976 & 0.237494 & 19.2 \\
\hline s_lo_hi & 30963.36 & 28002.32 & 10.57 & 13023.25 & 11163.52 & 16.65 & 0.440449 & 0.427074 & 3.13 \\
\hline s_hi_lo & 13289.39 & 12015.36 & 10.61 & 6285.19 & 6016.54 & 4.46 & 0.204667 & 0.168483 & 21.5 \\
\hline s_hi_hi & 30964.36 & 28053.28 & 10.37 & 13016.89 & 12732.82 & 2.23 & 0.370017 & 0.340823 & 8.56 \\
\hline Average & & & 10.28 & & & 6.83 & & & 5.61 \\
\hline
\end{tabular}

Table 2. Performance comparison of CHIDPSO with HIDPSO. 
Table 3. Performance comparison of CHIDPSO with NSHIDPSO.

\begin{tabular}{|c|c|c|c|c|c|c|c|c|c|}
\hline \multirow[b]{2}{*}{ ETC instances } & \multicolumn{3}{|c|}{ Makespan } & \multicolumn{3}{|c|}{ Mean flow time } & \multicolumn{3}{|c|}{ Reliability cost } \\
\hline & CHIDPSO & NSHIDPSO & EPD & CHIDPSO & NSHIDPSO & EPD & CHIDPSO & NSHIDPSO & EPD \\
\hline c_lo_lo & 13123.42 & 13575.71 & 3.44 & 6140.413 & 7057.59 & 14.93 & 0.186019 & 0.203629 & 9.46 \\
\hline c_lo_hi & 25201.01 & 29532.34 & 17.18 & 11638.44 & 14899.98 & 28.02 & 0.460741 & 0.456905 & -0.83 \\
\hline c_hi_lo & 11096.34 & 14038.69 & 26.51 & 5962.06 & 6113.44 & 2.53 & 0.181204 & 0.186552 & 2.95 \\
\hline c_hi_hi & 17103.05 & 30154.79 & 76.31 & 12002.87 & 14212.73 & 18.41 & 0.464482 & 0.459649 & -1.04 \\
\hline i_lo_lo & 25101.27 & 29387.81 & 17.07 & 13162.99 & 13567.78 & 3.07 & 0.305049 & 0.439342 & 44.02 \\
\hline i_lo_hi & 28056.91 & 31201.97 & 11.21 & 14008.09 & 15526.79 & 10.84 & 0.434033 & 0.478627 & 10.27 \\
\hline i_hi_lo & 25072.86 & 28983.85 & 25.61 & 11322.52 & 12598.62 & 11.27 & 0.393784 & 0.406979 & 3.35 \\
\hline i_hi_hi & 28045.24 & 31893.02 & 13.72 & 12072.92 & 15134.51 & 25.35 & 0.423777 & 0.479243 & 13.08 \\
\hline S_lo_lo & 23106.64 & 25233.79 & 9.21 & 10000.69 & 12013.17 & 20.12 & 0.237494 & 0.371189 & 56.29 \\
\hline s_lo_hi & 28002.32 & 30964.19 & 10.57 & 11163.52 & 15201.51 & 36.17 & 0.427074 & 0.475745 & 11.39 \\
\hline s_hi_lo & 12015.36 & 25278.29 & 110.3 & 6016.54 & 10801.23 & 79.52 & 0.168483 & 0.343588 & 103.9 \\
\hline s_hi_hi & 28053.28 & 31780.99 & 13.28 & 12732.82 & 14364.17 & 12.81 & 0.340823 & 0.472012 & 38.49 \\
\hline Average & & & 27.87 & & & 21.92 & & & 24.28 \\
\hline
\end{tabular}

objectives is impossible in case of Pareto dominance approach. It is inferred from table 3, the reference set and aggregating approach in CHIDPSO provided better objective values than NSHIDPSO in most of the ETC instances. The average overall improvements of CHIDPSO compared with NSHIDPSO with respect to Makespan: $27.87 \%$; Mean flow time: $21.92 \%$; Reliability Cost: $24.28 \%$ across all ETC instances.

The numerical results of the algorithms are validated using hypothesis test. Here, the wilcoxon signed-rank test [16] has been used to statistically compare the performance of the proposed algorithms. This test is performed and the results are presented in table 4 . In this test, it is essential that the following two parameters need to be set:

- Null hypothesis $\left(\boldsymbol{H}_{0}\right)$ is set to "No difference between two algorithms (CHIDPSO and HIDPSO)"
- Alternate hypothesis $\left(\boldsymbol{H}_{\boldsymbol{1}}\right)$ is set to "CHIDPSO performs better than the HIDPSO".

From table 4, the obtained wilcoxon test statistic $\mathrm{W}$ is 78 , which is greater than the upper critical value obtained from the wilcoxon signed rank test table [16]. Hence, $\mathrm{H}_{0}$ stands rejected and $\mathrm{H}_{1}$ accepted. Thus, there is an evidence; at the 5\% level of significance, that the proposed CHIDPSO performs better than HIDPSO.

The performance of CHIDPSO, HIDPSO and IDPSO are tested with large datasets. Table 5 presents the different ETC dimensions ( $\mathrm{n} \times \mathrm{m}$ matrix; where $\mathrm{n}$ is the number of tasks; $m$ is the number of processors) such as $1024 \times$ $32 ; 4096 \times 128$ and $8192 \times 256$.

The fitness values of the algorithms are calculated using Eq. (5), and the values with respect to different ETC dimensions $1024 \times 32$ and $4096 \times 128$ are given in table 6 and $8192 \times 256$ in table 7 .

Table 4. Statistical comparison of fitness value of CHIDPSO with HIDPSO.

\begin{tabular}{|c|c|c|c|c|c|c|c|}
\hline $\begin{array}{l}\text { ETC } \\
\text { Instance }\end{array}$ & $\begin{array}{l}\text { HIDPSO } \\
\text { (B) }\end{array}$ & $\begin{array}{l}\text { CHIDPSO } \\
\text { (A) }\end{array}$ & $\begin{array}{l}\text { Diff. } \\
\text { (B-A) }\end{array}$ & A. Diff. & O. Diff. & $\mathrm{R}$. & S.R. \\
\hline c_lo_lo & 7971.01 & 7705.57 & 265.44 & 265.44 & 265.44 & 1 & 1 \\
\hline c_lo_hi & 17877.11 & 14735.87 & 3141.24 & 3141.24 & 990.11 & 2 & 2 \\
\hline c_hi_lo & 7905.94 & 6823.39 & 1082.54 & 1082.54 & 1082.54 & 3 & 3 \\
\hline c_hi_hi & 13838.52 & 11642.46 & 2196.06 & 2196.06 & 1137.07 & 4 & 4 \\
\hline i_lo_lo & 16295.88 & 15305.77 & 990.11 & 990.11 & 1290.95 & 5 & 5 \\
\hline i_lo_hi & 18652.97 & 16826.09 & 1826.88 & 1826.88 & 1826.88 & 6 & 6 \\
\hline i_hi_lo & 16059.66 & 13758.23 & 2301.43 & 2301.43 & 2046.06 & 7 & 7 \\
\hline i_hi_hi & 18284.15 & 16047.35 & 2236.8 & 2236.8 & 2196.06 & 8 & 8 \\
\hline s_lo_lo & 14533.93 & 13242.98 & 1290.95 & 1290.95 & 2236.8 & 9 & 9 \\
\hline s_lo_hi & 18446.73 & 15666.42 & 2780.31 & 2780.31 & 2301.43 & 10 & 10 \\
\hline s_hi_lo & 8349.87 & 7212.794 & 1137.07 & 1137.07 & 2780.31 & 11 & 11 \\
\hline \multirow[t]{3}{*}{ s_hi_hi } & 18360.57 & 16314.51 & 2046.06 & 2046.06 & 3141.24 & 12 & 12 \\
\hline & & & & & & $\mathrm{W}+$ & 78 \\
\hline & & & & & & $\mathrm{W}-$ & - \\
\hline
\end{tabular}

*Diff. - Difference; A. Diff. - Absolute Difference; O. Diff. - Ordered Difference; R. - Rank; S.R.-Signed Rank 
Table 5. ETC dimension details.

\begin{tabular}{lcc}
\hline ETC dimension & Number of tasks & Number of processors \\
\hline $1024 \times 32$ & 1024 & 32 \\
$4096 \times 128$ & 4096 & 128 \\
$8192 \times 256$ & 8192 & 256 \\
\hline
\end{tabular}

From tables 6 to 7 , it can be observed that the proposed CHIDPSO provides better results than the both HIDPSO and IDPSO algorithms in all the ETC instances. Therefore, the proposed CHIDPSO provides better performance in the large ETC instances also.

The performance improvements of the fitness values of the CHIDPSO in terms of RPD values are given below:

- $1024 \times 32$ : Improvements of CHIDPSO are $8.21 \%$ and $16.74 \%$ compared with HIDPSO and IDPSO, respectively.

- $4096 \times 128$ : Improvements of CHIDPSO are $6.07 \%$ and $12.60 \%$ compared with HIDPSO and IDPSO, respectively.

- 8192 × 256: Improvements of CHIDPSO are $6.40 \%$ and $12.12 \%$ compared with HIDPSO and IDPSO, respectively.

All the simulation results with different ETC dimensions and the statistical test seem to indicate that the proposed CHIDPSO has shown a significant improvement when compared to CSA, IDPSO, HIDPSO, and NSHIDPSO algorithms for meta-task scheduling problem in distributed systems.

\section{Computational complexity}

This section presents the computation complexity [24] of the proposed CHIDPSO. The complexity analysis of the algorithm is explained in table 8 .
Table 7. Fitness value comparison of the algorithms in terms of $8192 \times 256$ ETC instances.

\begin{tabular}{lcccc}
\hline ETC & & & & \\
instance & CHIDPSO & HIDPSO & CHIDPSO & IDPSO \\
\hline c_lo_lo & $\mathbf{1 6 8 . 9 7}$ & 178.97 & $\mathbf{1 6 8 . 9 7}$ & 189.23 \\
c_lo_hi & $\mathbf{1 7 0 0 . 9 2}$ & 1727.92 & $\mathbf{1 7 0 0 . 9 2}$ & 1818.51 \\
c_hi_lo & $\mathbf{1 6 7 1 0 8 4 . 9}$ & 1771084.91 & $\mathbf{1 6 7 1 0 8 4 . 9}$ & 1878612.51 \\
c_hi_hi & $\mathbf{1 . 6 3 E + 0 7}$ & $1.73 \mathrm{E}+07$ & $\mathbf{1 . 6 3 E}+\mathbf{0 7}$ & $1.88 \mathrm{E}+07$ \\
i_lo_lo & $\mathbf{4 5 7 . 1 3}$ & 467.13 & $\mathbf{4 5 7 . 1 3}$ & 486.57 \\
i_lo_hi & $\mathbf{4 3 9 4 . 0 4}$ & 4694.04 & $\mathbf{4 3 9 4 . 0 4}$ & 4838.33 \\
i_hi_lo & $\mathbf{4 3 6 2 2 9 1 . 5}$ & 4662291.51 & $\mathbf{4 3 6 2 2 9 1 . 5}$ & 4804507.51 \\
i_hi_hi & $\mathbf{4 . 4 1 E + 0 7}$ & $4.71 \mathrm{E}+07$ & $\mathbf{4 . 4 1 E}+\mathbf{0 7}$ & $4.91 \mathrm{E}+07$ \\
s_lo_lo & $\mathbf{6 6 . 6 3}$ & 75.63 & $\mathbf{6 6 . 6 3}$ & 80.73 \\
s_lo_hi & $\mathbf{7 1 0 . 8 8}$ & 760.88 & $\mathbf{7 1 0 . 8 8}$ & 791.62 \\
s_hi_lo & $\mathbf{7 1 4 7 2 8 . 2 1}$ & 774728.21 & $\mathbf{7 1 4 7 2 8 . 2 1}$ & 828071.31 \\
s_hi_hi & $\mathbf{7 1 6 5 2 0 1 . 5}$ & 7565201.51 & $\mathbf{7 1 6 5 2 0 1 . 5}$ & 8071759.51 \\
\hline
\end{tabular}

The looping variables involved in the algorithm are $\mathbf{N}$ : Swarm size; n: Size of the particle; m: Number of processors; G: Total number of iterations and the additional variable $\mathrm{k}$ is used to limit the creation of sub sets in SG method in reference set updation phase.

The six phases in CHIDPSO are Swarm initialization, Particle evaluation, Update Pbest, Find Lbest, Update reference set and Particle updation. These phases are executed in $G$ number of times. The Reference set updation takes more time for execution when compared to other phases. Therefore, the worst case time complexity of CHIDPSO is $\mathrm{O}\left(\mathrm{n}^{2}\right)$. Table 9 presents the comparison of complexity of the proposed algorithm with other algorithms.

From table 9, it is inferred that the complexity of CHIDPSO is lesser when compared to the existing algorithms such as NSGA-II, NSPSO and NSHIDPSO. The CHIDPSO and the existing CSA have same time complexities. The complexity of CHIDPSO is slightly higher than IDPSO and HIDPSO. However, the simulation results and statistical test proved that the proposed CHIDPSO performs better than IDPSO and HIDPSO algorithms.

Table 6. Fitness value comparison of the algorithms in terms of different ETC instances.

\begin{tabular}{|c|c|c|c|c|c|c|c|c|c|}
\hline $\begin{array}{l}1024 \times 32 \\
\text { ETC Instance }\end{array}$ & CHIDPSO & HIDPSO & CHIDPSO & IDPSO & $\begin{array}{c}4096 \times 128 \\
\text { ETC Instance }\end{array}$ & CHIDPSO & HIDPSO & CHIDPSO & IDPSO \\
\hline c_lo_lo & 200.61 & 230.22 & 200.61 & 249.22 & c_lo_lo & 174.41 & 186.03 & 174.41 & 199.46 \\
\hline c_lo_hi & 2006.41 & 2182.51 & 2006.41 & 2358.57 & c_lo_hi & 1801.54 & 1858.45 & 1801.54 & 1998.55 \\
\hline c_hi_lo & 2000830 & 2260501 & 2000830 & 2591563.51 & c_hi_lo & 1800805 & 1874492.21 & 1800805 & 1993136.9 \\
\hline c_hi_hi & $2.07 E+07$ & $2.30 \mathrm{E}+07$ & $2.07 E+07$ & $2.50 \mathrm{E}+07$ & c_hi_hi & $1.71 E+07$ & $1.89 \mathrm{E}+07$ & $1.71 E+07$ & $1.99 \mathrm{E}+07$ \\
\hline i_lo_lo & 400.97 & 432.49 & 400.97 & 467.47 & i_lo_lo & 437.51 & 465.85 & 437.51 & 491.17 \\
\hline i_lo_hi & 4001.79 & 4365.46 & 4001.79 & 4666.17 & i_lo_hi & 4200.75 & 4584.41 & 4200.75 & 4863.46 \\
\hline i_hi_lo & 4001565 & 4362632 & 4001565 & 4679823.51 & i_hi_lo & 4359621 & 4653622.51 & 4359621 & 4831489.5 \\
\hline i_hi_hi & $4.07 E+07$ & $4.44 \mathrm{E}+07$ & $4.07 E+07$ & $4.73 \mathrm{E}+07$ & i_hi_hi & $4.30 E+07$ & $4.67 \mathrm{E}+07$ & $4.30 \mathrm{E}+07$ & $4.87 \mathrm{E}+07$ \\
\hline s_lo_lo & 401.80 & 419.47 & 401.80 & 455.13 & s_lo_lo & 407.60 & 426.69 & 407.60 & 454.87 \\
\hline s_lo_hi & 4000.13 & 4083.69 & 4000.13 & 4202.65 & s_lo_hi & 4092.41 & 4229.45 & 4092.41 & 4426.59 \\
\hline s_hi_lo & 4001119 & 4208786 & 4001119 & 4477223.51 & s_hi_lo & 4064645 & 4245952 & 4064645 & 4572696 \\
\hline s_hi_hi & $4.00 E+07$ & $4.17 \mathrm{E}+07$ & $4.00 E+07$ & $4.49 \mathrm{E}+07$ & s_hi_hi & $4.02 E+07$ & $4.22 \mathrm{E}+07$ & $4.02 E+07$ & $4.58 \mathrm{E}+07$ \\
\hline
\end{tabular}


Table 8. Asymptotic time complexity of CHIDPSO algorithm.

\begin{tabular}{lc}
\hline Phase name & $\begin{array}{r}\text { Asymptotic } \\
\text { complexity }\end{array}$ \\
\hline 1) Swarm initialization & $\mathrm{O}(\mathrm{N} \mathrm{n} \mathrm{m})$ \\
i) Particle initialization: $\mathrm{O}(\mathrm{n})$ & \\
ii) Processor allocation: $\mathrm{O}(\mathrm{N} \mathrm{n} \mathrm{m)}$ & $\mathrm{O}(\mathrm{N})$ \\
2) Particle Evaluation & $\mathrm{O}(\mathrm{N})$ \\
3) Update Pbest & $\mathrm{O}(\mathrm{N} \mathrm{n})$ \\
4) Find Lbest & $\mathrm{O}\left(\mathrm{n}^{2}\right)$ \\
5) Update reference set & \\
i) $\mathrm{O}\left(\mathrm{n}^{2}\right)-$ Subset Generation Method & \\
ii) O(SCSize $)$ - Solution Combination & $\mathrm{O}(\mathrm{N})$ \\
The maximum time complexity is $\mathrm{O}\left(\mathrm{n}^{2}\right)$ & \\
6) Particle Updation &
\end{tabular}

Table 9. Complexity comparison of CHIDPSO algorithm with other algorithms.

\begin{tabular}{lc}
\hline Name of the algorithm & $\begin{array}{l}\text { Asymptotic } \\
\text { complexity }\end{array}$ \\
\hline HIDPSO and IDPSO [16] & $\mathrm{O}(\mathrm{N} \mathrm{n} \mathrm{m})$ \\
NSGA-II, NSPSO and NSHIDPSO [22] & $\mathrm{O}\left(\mathrm{M} \mathrm{N}^{2}\right)$ \\
CSA and CHIDPSO & $\mathrm{O}\left(\mathrm{n}^{2}\right)$ \\
\hline
\end{tabular}

\section{Conclusion}

Task Scheduling (TS) is a well-known discrete optimization problem, which is a main demanding issue in distributed systems. PSO is a well-known meta-heuristic algorithm for solving the scheduling problem. CSA is an improved version of PSO, which follows Gbest model for particles communication. The benefit of using the CSA is a reference set model. To reduce the premature convergence problem in CSA and utilize the advantages of CSA, the CHIDPSO was proposed and presented in this paper. This is a hybridization of HIDPSO and CSA. The efficiency of the algorithm is tested using the performance metrics such as makespan, mean flow time, reliability cost, fitness and resource utilization. The performance improvements of the proposed algorithm with other algorithms are compared based on the RPD metric. The incorporation of heuristic based initialization, BHT based particle communication and reference set in CHIDPSO resulted in reduced premature convergence problem and improved the diversity of the algorithm. The simulation results and hypothesis test confirmed that the CHIDPSO algorithm provided better performance than CSA, IDPSO, HIDPSO, and NSHIDPSO algorithms for meta-task scheduling problem in distributed systems. Future work of the research focuses on evaluating the efficiency of the CHIDPSO algorithm in dynamic and preemptive scheduling environment.

\section{References}

[1] Kennedy J and Eberhart R 1995 Particle swarm optimization. In: Proceeding of the Fourth IEEE International Conference on Neural Networks, vol. 4(1), pp. 1942-1948

[2] Braun T D, Siegel H J and Bec N 2001 A comparison of eleven static heuristics for mapping a class of independent tasks onto heterogeneous distributed computing systems. $J$. Parallel Distrib. Comput. 61(1): 810-837

[3] Izakian H, Abraham A and Snasel V 2009 Performance comparison of six efficient pure heuristics for scheduling meta-tasks on heterogeneous distributed environments. Neural Netw. World 19 (6): 695-710

[4] Abraham A, Liu H, Grosan C and Xhafa F 2008 Nature inspired meta-heuristics for grid scheduling: single and multi-objective optimization approaches. Metaheuristics for Scheduling in Distributed Computing Environments 146(1): 247-272

[5] Kang Q and He H 2011 A novel discrete particle swarm optimization algorithm for meta-task assignment in heterogeneous computing systems. Microprocess. Microsyst. 35(1): $10-17$

[6] Sarathambekai S and Umamaheswari K 2014 Task scheduling in distributed systems using discrete particle swarm optimization. Int. J. Adv. Res. Comput. Sci. Softw. Eng. 4(2): 510-522

[7] Xin J, Chen G and Hai Y 2009 A particle swarm optimizer with multistage linearly-decreasing inertia weight. In: Proceeding of IEEE International Joint Conference on Computational Sciences and Optimization, vol. 1(1), pp. 505-508

[8] Bansal J C, Singh P K, Saraswat M, Verma A, Jadon S S and Abraham A 2011 Inertia weight strategies in particle swarm optimization. In: IEEE World Congress on Nature and Biologically Inspired Computing (NaBIC), vol. 3 (1), pp. 633-640

[9] Sarathambekai S and Umamaheswari K 2013 Comparison among four modified discrete particle swarm optimization for task scheduling in heterogeneous computing systems. Int. J. Soft Comput. Eng. (IJSCE) 3(2): 371-378

[10] Sarathambekai S and Umamaheswari K 2016 Task scheduling using multi-objective particle swarm optimization with hamming inertia weight. Adv. Intell. Syst. Comput. 2(1): 57-65

[11] Sarathambekai S and Umamaheswari K 2016 Task scheduling using multi-objective hamming discrete particle swarm optimisation in distributed systems. Int. J. Swarm Intell. 2(2/3/4): 100-116

[12] Sarathambekai S and Umamaheswari K 2017 Task scheduling using hamming particle swarm optimization in distributed systems. J. Comput. Inform. 36(1): 1001-1021

[13] Yin P-Y, Glover F, Laguna M and Zhu J-X 2010 Cyber swarm algorithms-improving particle swarm optimization using adaptive memory strategies. Eur. J. Oper. Res. 201(1): 377-389

[14] Yin P-Y and Su E-P 2011 Cyber Swarm optimization for general keyboard arrangement problem. Int. J. Ind. Ergon. 41(2): 43-52

[15] Yin P-Y and Chiang Y-T 2013 Cyber swarm algorithms for multi-objective nurse rostering problem. Int. J. Innov. Comput. Inf. Control 9(5): 2043-2063

[16] Sarathambekai S and Umamaheswari K 2017 Task scheduling in distributed systems using heap intelligent discrete particle swarm optimization. Int. J. Comput. Intell. 33(4): 737-770 
[17] Sarathambekai S and Umamaheswari K 2016 Intelligent discrete particle swarm optimization for multiprocessor task scheduling problem. J. Algorithms Comput. Technol. 2(1): 1-10

[18] Qin X and Jiang H 2001 Dynamic, reliability-driven scheduling of parallel real-time jobs in heterogeneous systems. IEEE Int. Conf. Parallel Process. 2(1): 113-122

[19] Bahman Naderi and Rubén Ruiz 2014 A scatter search algorithm for the distributed permutation flow shop scheduling problem. Eur. J. Oper. Res. 239(1): 323-334

[20] Ali S and Siegel H J 2000 Representing task and machine heterogeneities for heterogeneous computing systems. Tamkang J. Sci. Eng. 3 (3): 195-207
[21] Fahd Alharbi 2012 Simple scheduling algorithm with load balancing for grid computing. Asian Trans. Comput. 2(2): $8-15$

[22] Sarathambekai S and Umamaheswari K 2018 Multi-objective optimization techniques for task scheduling problem in distributed systems. Comput. J. 61(2): 248-263

[23] Sarathambekai S and Umamaheswari K 2017 Performance comparison of discrete particle swarm optimisation and shuffled frog leaping algorithm in multiprocessor task scheduling problem. Int. J. Adv. Intell. Paradig. 9(2/3): 139-163

[24] Turky A M and Abdullah S 2014 A multi-population harmony search algorithm with external archive for dynamic optimization problems. Inf. Sci. 272(2): 84-95 